\title{
DESAFÍOS DE LA POLÍTICA DE SALUD MENTAL SUSTITUTIVA A LO MANICOMIAL: QUE ABRIR PARA CERRAR. CERRAR EL MANICOMIO NO ES ACHICAR EL ESTADO
}

Verónica Almeida
ATE, Rosário Argentina; 1veroalmeida@gmail.com

Mariana Chidichimo

ATE, Rosário Argentina; marianachidichimo@hotmail.com

Laura Coll

coll_laura@hotmail.com

Florencia Orpinell

ATE, Rosário Argentina; florenciaorpinell@gmail.com

Celina Pochettino

ATE, Rosário Argentina; celinapochettino@hotmail.com

Laura Valdano

ATE, Rosário Argentina; Ivaldano@yahoo.com.ar

Informações do artigo

Recebido em 15/08/2017

Aceito em 20/12/2017

\begin{abstract}
Resumo
Debater "diagnósticos e desafios" atuais desde a perspectiva dos trabalhadores implica uma leitura transversal das práticas no campo da saúde mental e o conflito que se gera na inevitável tensão entre estas e o desenho das políticas. Abordar o fechamento do manicômio é prioritário para avançar no modelo de atenção com perspectiva de direitos que garanta condições aos processos de assistência em saúde mental. Levando em conta o avanço dos processos de reforma no nosso país e na região, falar em fechamento coloca-nos, hoje, numa encruzilhada. Devemos ter cautela e, ao mesmo tempo, audácia. Hoje, falar em fechamento coagula o sentido neoliberal do afastamento do Estado como garante de direitos. Por isto, dizemos "Fechar o manicômio não é diminuir o Estado". Trata-se de abrir e exigir o desenho de uma política em saúde mental que substitua o manicômio sem gerar desassistência.

Palavras-chave: Fechamento do manicômio. Privatização do Estado. Trabalhadores. Políticas Públicas.
\end{abstract}

\section{¿QUIÉNES SOMOS?}

No se puede cambiar con una ley un paradigma, el pensamiento de los psiquiatras.
El proceso de desinstitucionalización, (...) no es solamente cerrar los manicomios
(...) es cambiar el paradigma, la mirada que hay sobre la locura, sobre las
necesidades de la gente, (...) Nosotros hablamos de desinstitucionalizar el hospital
psiquiátrico, pero también la psiquiatría y la locura y la relación con la locura (ROTELLI, 2014).

Representamos un colectivo de trabajadoras organizadas en un espacio sindical (ATE), realizamos nuestras prácticas en salud mental desde una perspectiva política y clínica. Perspectiva que no separa las condiciones de trabajo de las condiciones de asistencia. Venimos de un proceso de organización histórico que nos trasciende y que además es federal. Tenemos una lectura crítica de la dinámica que adquiere el diseño de las políticas sociales y los conflictos, impasses, dificultades que se desprenden en el proceso mismo de trabajo. 
Los miembros de este colectivo trabajamos en diferentes instituciones y dispositivos dependientes de la Dirección de salud mental de la Provincia de Santa Fe, en la ciudad de Rosario, Argentina. Nuestro análisis intenta trascender la suma de cada uno de los espacios de trabajo. Intentamos construir una lectura integral de la política en salud mental tensando nuestras propias experiencias con nuestra posición en torno a las Políticas Públicas. Desde nuestro lugar de trabajadoras, se trata de construir una lectura transversal de las prácticas que se desarrollan en nuestro campo y el conflicto que se genera en la tensión inevitable entre éstas y el diseño de las políticas.

En un documento diagnóstico que elaboramos a finales de 2015, ubicábamos la necesidad de abordar como problema principal el cierre del manicomio, para avanzar en el diseño de un modelo de atención en perspectiva de derechos que garantice condiciones dignas a los procesos de asistencia en salud mental; entendiendo por esto la accesibilidad, la continuidad del proceso de atención en el circuito de la red asistencial, la creación de una efectiva red de servicios intersectoriales que permitan tanto el abordaje de las problemáticas vinculadas al padecimiento subjetivo en espacios territoriales público estatales, como una inclusión laboral formal, políticas concretas de vivienda; en el marco de contar con condiciones de trabajo para lograr condiciones de asistencia en la perspectiva del paradigma comunitario.

El horizonte actual de la legislación argentina, luego de la sanción de la Ley Nacional de Salud Mental y Adicciones No 26.657 (en adelante LNSMyA) en el año 2010, establece el fin de los manicomios para el año 2020 y la obligación de abrir simultáneamente instituciones público estatales para garantizar la continuidad del proceso de atención para las personas hoy internadas y acceso a la atención en salud mental para la población. Esto implica el desafío de diseñar un modelo de atención que prescinda del manicomio y sus prácticas. Entendiendo que el manicomio es una lógica y no solo las instituciones monovalentes de encierro.

A más de seis años de su sanción, la LNSMyA sigue sin ser implementada en sus aspectos más cruciales y necesarios. La LNSMyA propone tanto un cambio de paradigma respecto del reconocimiento de las personas con padecimiento psíquico como sujetos de derechos partiendo de la presunción de capacidad, como un modelo asistencial comunitario que signa normativamente el fin del modelo manicomial institucionalista. Aunque el avance que plantea la Ley no es suficiente para lograr una sustitución efectiva, marca el campo auspiciosamente. El artículo 11 de la 26.657 señala a las políticas públicas pendientes el rumbo de lo sustitutivo. 
Art. 11. - La Autoridad de Aplicación debe promover que las autoridades de salud de cada jurisdicción, en coordinación con las áreas de educación, desarrollo social, trabajo y otras que correspondan, implementen acciones de inclusión social, laboral y de atención en salud mental comunitaria. Se debe promover el desarrollo de dispositivos tales como: consultas ambulatorias; servicios de inclusión social y laboral para personas después del alta institucional; atención domiciliaria supervisada y apoyo a las personas y grupos familiares y comunitarios; servicios para la promoción y prevención en salud mental, así como otras prestaciones tales como casas de convivencia, hospitales de día, cooperativas de trabajo, centros de capacitación socio-laboral, emprendimientos sociales, hogares y familias sustitutas. (2010)

De modo disímil en cada provincia o región se han ido creando dispositivos o servicios alternativos, que no logran asistir las principales demandas en salud mental, que demuestran ausencia de planificación y desarticulación con la red de servicios del sistema de salud. Por tal motivo no han logrado impactar directamente en la lógica manicomial, ni en las transformaciones de las instituciones monovalentes, con el riesgo de que se instituya un modelo mixto de atención, que avale la constante derivación al sector privado (instituciones fundamentalmente de encierro) de las problemáticas principales que padece la población que accede al sistema público de salud.

Durante el 2016 -cambio de gobierno mediante- el Estado nacional, vía la autoridad de aplicación, se posicionó como foco resistencial a los procesos iniciados con la ley $\mathrm{N}^{\circ} 26657$, afirmando formalmente cumplir con la ley, al tiempo que se invalidan los instrumentos o instancias creadas por la misma.

Algunos de los retrocesos fueron la no convocatoria al Consejo Consultivo Honorario en Salud Mental y Adicciones (CCH) limitando así la participación comunitaria en la toma de decisiones en políticas públicas, y a la Comisión Nacional Interministerial en Políticas de Salud Mental y Adicciones (CONISMA), la que a su vez fue trasladada desde la Jefatura de Gabinete de Ministros a la órbita del Ministerio de Salud -primera modificación al decreto reglamentario de la LNSMyA-, con la pérdida del carácter intersectorial (Ferreira y Stolkiner, 2017)

Esta situación se agrava aún más a partir de la reciente resolución № 1061-E/2017 dictada por el Ministerio de Salud de la Nación en agosto del corriente año, con la cual se modifica la composición actual del Consejo Consultivo Honorario en Salud Mental y Adicciones, integrado por 30 organizaciones de reconocida trayectoria, a cambio de un supuesto equipo crítico e integrado por "expertos", los cuales serán seleccionados a discrecionalmente por el Director de Salud Mental André Blake, atentándose contra una de las ideas centrales de 
la legislación vigente referida a la participación comunitaria en la planificación y toma de decisiones en la política en salud mental.

A modo de resistencia a las embestidas del nuevo gobierno, las organizaciones del campo de la salud mental impulsamos la creación del Movimiento Nacional en Defensa de la ley de Salud Mental motivados por el avasallamiento que constituyó la derogación de la Resolución 1484/15, la cual se refería a la habilitación de instituciones de salud incluyendo dispositivos intermedios y establecía plazos claros para la adecuación y cierre de instituciones monovalentes. En su lugar se sanciona la Resolución 1876/16 la cual entre otras medidas regresivas avala la práctica de electroshock y recentra la disciplina médico-psiquiátrica por sobre el resto de las que componen el campo de la salud mental, tendiendo así al quiebre de la interdisciplina (CONISMA, 2016)

En este contexto de retrocesos en nuestro país y en la región se suma el decreto de necesidad y urgencia del presidente Macri DNU No 908/16 acerca de la implementación de la Cobertura Universal en Salud (CUS) tendiente, a pesar de la nominación, a coartar el acceso universal a la salud.

\section{Encrucijadas y trampas de un sistema universal de salud}

A los fines de justificar la creación de un Decreto de Necesidad y Urgencia (DNU 908/2016) se argumenta que existe "una circunstancia extraordinaria que hace imposible seguir los trámites de rigor previstos por la Constitución Nacional para la sanción de las leyes".

Siguiendo el Documento del Movimiento por el Derecho a la Salud entendemos, que con un DNU se pretende eludir una discusión parlamentaria y mecanismos de participación popular como la Audiencia Pública para un problema que aparece como una urgencia actual, cuando en realidad data de décadas. (IDEP, 2017)

La Organización Mundial de la Salud en su informe sobre la salud en el mundo: "La financiación de los Sistemas de Salud. El camino hacia la cobertura universal" declara que:

La cobertura sanitaria universal (CSU) implica que todas las personas y comunidades reciban los servicios de salud que necesitan sin tener que pasar penurias financieras para pagarlos. Abarca toda la gama de servicios de salud esenciales de calidad, desde la promoción de la salud hasta la prevención, el tratamiento, la rehabilitación y los cuidados paliativos. 
La CSU permite a todos acceder a servicios que atienden las causas más importantes de las enfermedades y la muerte, y asegura que la calidad de esos servicios sea suficientemente buena para mejorar la salud de las personas que los reciben (OMS, 2010)

La propuesta de un sistema universal de salud podría concebirse como una herramienta poderosa en el ámbito de la salud pública, en tanto comprende dos aspectos fundamentales: por un lado, el acceso a los servicios de buena calidad necesarios para lograr que cada persona y comunidad tengan buena salud, incluidas la promoción, la prevención, el tratamiento, la rehabilitación y los cuidados paliativos o la atención a largo plazo, junto con las medidas necesarias para abordar los determinantes de la salud; y por el otro, los mecanismos, las políticas y los reglamentos financieros requeridos para garantizar la protección financiera y evitar que la mala salud conduzca a la pobreza o la empeore. Sin embargo en nuestro país no se toman los aspectos positivos de estas definiciones de la OMS sino los aspectos negativos, los que permiten pensarlas en términos de mercado.

Historizando el concepto de Cobertura Universal de Salud (CUS) podemos analizar que se inscribe en una tradición que se remonta en su origen a la segunda posguerra y más específicamente a la Declaración Universal de los Derechos del Hombre.

En su documento fundacional (1948) la OMS proclama en su artículo 2: "El goce del grado máximo de salud que se pueda lograr es uno de los derechos fundamentales de todo ser humano sin distinción de raza, religión, ideología política o condición económica o social"

Posteriormente, en la Declaración de Alma Ata (1978) la OMS y los países que suscriben realizan una nueva definición política cuando enuncian en el artículo 5:

la atención primaria de salud es la clave para alcanzar esa meta como parte del desarrollo conforme al espíritu de la justicia social"; y en el artículo 6 define a la Atención Primaria de la Salud como "la asistencia sanitaria esencial basada en métodos y tecnologías prácticos, científicamente fundados y socialmente aceptables, puesta al alcance de todos los individuos y familias de la comunidad, mediante su plena participación, y a un costo que la comunidad y el país puedan soportar...

...A partir de ese momento la idea de "asistencia sanitaria esencial" y la de "prestaciones básicas" serán parte crucial de todos los documentos de la OMS sobre cobertura de salud". (MIGUELES, 2017)

La encrucijada la podemos ubicar en:

... la profunda contradicción que existe en el concepto de prestaciones básicas que la CUS tiene como uno de sus principios, y el derecho al grado máximo de salud de todos los ciudadanos, establecido en la Constitución de la OMS (MIGUELES, 2017) 
La concepción de la "canasta básica" tiene un fracasado antecedente en el documento del Banco Mundial en 1993 "Invertir en Salud", donde se critica el gasto inefectivo en salud y se propone la adopción de paquetes de medidas de salud pública y atención médica básica para los países en desarrollo.

Los sistemas gerenciados, los pagos de bolsillos en hospitales, eran otras propuestas del Banco. Todas estas estrategias que fueron aplicadas en los go en varios países del mundo, han demostrado su inefectividad e incluso una gran capacidad de daño, ya que en lo concreto las canastas básicas fueron restrictivas para prácticas médicas de probada efectividad y el gerenciamiento de los servicios se basó en la subprestación para aumentar los márgenes de ganancia. Por ello pensamos que esta "canasta básica" de la CUS tiende a excluir prácticas de salud que hoy se efectúan con buenos resultados, limitando derechos.

(...) Con esta idea de paquetes de servicios "la OMS parece haber claudicado de sus principios iniciales, aduciendo a limitaciones económicas, aquello que en realidad se debe al juego político en la distribución de los recursos; de esta forma se tiende a acrecentar las fuerzas estratificantes del modo de producción capitalista (MIGUELES, 2017)

En nuestro país el sistema de salud quedó conformado desde hace seis décadas de forma tal que sus rasgos son: la débil regulación estatal, la fragmentación, las concentraciones coorporativas, la desigual distribución, la desproporción entre la inversión en tecnologías y los resultados obtenidos. Todo ello consolidó y profundizó inequidades regionales que a pesar de los "diferentes" intentos de abordaje por los gobiernos de las últimas décadas sólo han mantenido y profundizado las características descriptas.

La salud ya es en la Argentina formalmente universal y gratuita para todos sus habitantes; es concebida como un derecho desde el año 1994 con la reforma de la Constitución Nacional donde se adhiere al tratado internacional de la "Declaración Universal de Derechos Humanos" (Art.75, inciso 22). Sin embargo, las medidas que el gobierno nacional ha introducido con el decreto CUS tienen precisamente una dirección inversa; se habla de Cobertura Universal y el propio decreto recorta la cobertura a quienes no tienen cobertura de las Obras sociales o seguros privados, por lo tanto, no será Universal

Dista, a su vez, de la propuesta inicial de la OMS basada en fondos mancomunados y retoma la idea de "canasta básica" marcando el avance de los mercados sobre el Derecho a la salud, es decir propiciando la mercantilización de la Salud.

¿Quién administra? ¿Qué modelo de atención se financia? ¿Qué sistema de salud se consolida? son preguntas que orientan nuestro recorrido y que necesitamos discutir y visibilizar hoy.

El anuncio de la CUS se sustenta en utilizar el Fondo de Redistribución de la Superintendencia de Servicios de Salud, de ese modo se transfiere por única vez un monto 
acotado 8000 millones de pesos, aproximadamente $2 \%$ del gasto total en salud, previendo el retiro progresivo del Estadonacional, transfiriendoa provinciasymunicipiosla responsabilidad, de modo que nuevamente asistimos a la descentralización de políticas sociales sin fondos, con el conocido riesgo de no poder sostenerse en el tiempo, la consecuente precarización de lo público estatal y la consolidación entonces del argumento para las privatizaciones: lo público estatal es precario, no funciona bien, es necesario acuerdos público-privados. Constituyendo esto una clara y planificada decisión de mercantilización de la salud.

Es decir, la profundización de la Reforma por medio de la implementación de la CUS, permitiría avanzar en la institucionalización de un sistema de aseguramiento que implica una lógica de "contrato" y no de "derecho", desconociendo que la Constitución Argentina garantiza el derecho a la salud a toda la población. Esta estrategia entiende a la salud como bien de consumo, y propone una reducción de los servicios y prestaciones que ya son garantizados por el actual sistema público de salud. Este paradigma que pretende instalar la idea de la salud como un gasto generará además una desigualdad en el acceso a la salud, ya que se medirá de acuerdo al ingreso económico de cada usuario, con una lógica de seguros individuales para algunas enfermedades.

Esto ya ha sucedido en los países que siguen las recetas impulsadas por los organismos de crédito (liderados por Banco Mundial y BID), así como por la OMS y OPS, que marcan la agenda de las Reformas del sector Salud en particular y del Estado en general para los países de la región.

Hoy asistimos a una avanzada concreta de la Reforma del Sistema de Salud que se inició en los gó y que no se desmontó en todos estos años. Por lo tanto, nos vemos obligados a advertir el engaño de discursos del campo de la salud que se pretenden progresistas, universalistas pero que son los que sostienen las políticas focales y privatistas. Es decir, enmascaran un achicamiento del Estado en nombre de principios que compartimos. Por eso decimos: Cerrar el manicomio no es achicar el Estado.

Creemos que todas las problemáticas específicas vinculadas a la SALUD y DDHH que pugnan por ser parte de las agendas de la gestión pública (precarización laboral, condiciones de trabajo, salario, Salud Mental, Producción Pública de Medicamentos, Salud de Género, políticas de infancia, etc.) serán sobrepasadas y arrastradas por esta estrategia. 


\section{Cerrar el manicomio no es achicar el estado. La encrucijada neoliberal y la audacia que requiere su cierre}

Nos preguntamos qué dispositivos o instituciones resulta necesario abrir para que cerrar el manicomio no termine siendo restringir derechos y achicar el Estado.

Dado el avance de los procesos de reforma en nuestro país y en la región, signada por las políticas de ajuste, achicamiento del Estado, recortes presupuestarios y eliminación de políticas sociales; hablar de cierre nos plantea hoy una encrucijada. Debemos ser cautelosos, cuidadosos y al mismo tiempo audaces. Hoy hablar de cierre coagula el sentido neoliberal del corrimiento del Estado como garante de derechos.

A modo de ejemplo en la ciudad de Rosario, el Centro Regional de Salud Mental "Dr. Agudo Avila", uno de los tres históricos hospitales monovalentes públicos, de la provincia de Santa Fe, en los últimos tres años, ha ido cerrando camas; según informes oficiales (Diagnose, 2017) 19 plazas figuran fuera de servicio.

Este dato de reducción de camas se convierte en un analizador de la problemática que queremos plantear; ya que podría ser auspicioso en relación a un proceso de transformación de los modos de asistencia; sin embargo, el destino de las mismas terminó siendo derivaciones a instituciones del ámbito de lo privado.

Estamos frente a una política de desasistencia y de transinstitucionalización, entendiendo por esto tanto, la transferencia de recursos del Estado hacia instituciones privadas con lógicas manicomiales, como la persistente derivación a la Colonia Psiquiátrica de Oliveros (CPO) - el otro hospital monovalente de la Provincia Santa Fe - ubicado territorialmente a 60 km de la ciudad de Rosario. Según datos del departamento de estadísticas de la CPO en el primer semestre del 2017 se recepcionaron 150 consultas, de las cuales 101 tuvieron criterio de internación y de éstas, 50 eran provenientes de la ciudad de Rosario.

La constante derivación hacia el sector privado es también una política recurrente tanto en las problemáticas de niñez, adolescencia, como en las de adicciones.

La complejidad que se presenta en el entrecruzamiento de vulneración de derechos y padecimientos psíquicos severos en la infancia y adolescencia encuentra en las políticas estatales actuales un contundente "no hay lugar", recrudeciéndose entonces las condiciones que producen desamparo, exclusión, padecimientos y muertes de estos niños/as y jóvenes. 
En nuestra práctica nos encontramos cada vez más con jóvenes arrasados por las condiciones de vida, cercenados por la muerte a cada paso, consumidos por sustancias tóxicas, siempre al borde de la muerte, del delito, de la autoflagelación. Cuerpos mutilados, cortados, rajados, expulsados de todos lados; con suerte alguna vez pusieron un pie en la escuela, pero es hoy un recuerdo vago. Niñas madres, sometidas y expropiadas de su propio cuerpo también; cada vez más mujeres adolescentes son desaparecidas y luego vendidas para su explotación sexual, siendo la policía actores principales de este negocio porque son las comisarías las primeras estaciones. Decenas de adolescentes mueren víctimas del narcotráfico y la violencia producida en estos circuitos ilegales que abundan. Cada vez son más la demanda de niños con desorganización subjetiva en condiciones de extrema pobreza y vulneración de todos sus derechos; arrasados por esta sociedad desigual.

Los rasgos, lo característico, lo propio de estas categorías (niñez y adolescencia), aparece borrado, desdibujado; a veces solo se trata de empezar a construir algo de esto. Por eso como trabajadoras del estado no nos quedamos en la queja o en la sola denuncia; sino que estamos permanentemente construyendo estrategias para abordar esta complejidad, poniendo el cuerpo, la imaginación, y la creatividad al servicio de esta población arrasada. En este contexto se hace necesario pensar con qué sujetos (en el más afortunado de los casos) nos estamos encontrando, cuáles son las características de estas nuevas subjetividades, de qué se tratan estos modos de padecimiento que constantemente ponen en jaque las teorías, las herramientas con las que contamos, o las instituciones mismas.

Entendiendo que el tiempo y el espacio son condiciones que lo subjetivo requiere para transitar un camino restitutivo; nos preguntamos qué instituciones o dispositivos necesitamos abrir para alojar estas demandas en salud mental.

Las internaciones en polivalentes por crisis subjetivas se realizan con mayor frecuencia en estos últimos años, sin embargo, no se ha aumentado el número de camas, ni la adecuación edilicia, ni la formación a los equipos de los efectores de los hospitales generales, para que estos procesos se realicen en condiciones dignas. Esto redunda en que muchas situaciones no acceden a un abordaje de salud desde la complejidad que requieren; o no es posible la continuidad del proceso asistencial por la ausencia de servicios intermedios.

La escasa capacitación de los equipos, así como la falta de espacios de retrabajo de las prácticas y los procesos; las condiciones edilicias, la precariedad de los trabajadores; y 
fundamentalmente la ausencia de lineamientos y circuitos del proceso asistencial deja a los equipos de los efectores en encerronas que complican la lógica de dicho proceso.

Entendemos que de ninguna manera la internación en los hospitales generales, en estas condiciones es suficiente y que permanecerá en la precariedad si sus equipos continúan sin contar con servicios comunitarios de salud mental en los territorios con los que puedan articular el proceso asistencial una vez transitada la crisis subjetiva.

La provincia de Santa Fe no cuenta con un plan provincial de salud mental. Este debería articularse en el diseño de un sistema de salud público integral fundado en perspectiva epidemiológica, es decir que contemple cuales son los problemas más incidentes de nuestra población.

Esto produce el interrogante de cómo se planifican las políticas en salud mental, de cómo se elaboran los objetivos y directrices, o en base a qué lectura epidemiológica se conforma cada dispositivo de salud mental sin un análisis respecto a cuáles son las principales demandas de atención, o las problemáticas más emergentes.

Entendemos como prioritario la elaboración de proyectos y directrices que consideren las dimensiones políticas e institucionales de las problemáticas y no desatiendan las dimensiones comunitarias y clínicas de las mismas.

Si bien existen, en la ciudad de Rosario, una serie de dispositivos llamados sustitutivos, como se describen en "Lineamientos prioritarios de la política de Salud Mental en la provincia de Santa Fe" (Augsburger AC, Gerlero SS, Taboada E. 2016) estos no logran conformar una red de asistencia de lógica sustitutiva a lo manicomial. Son fundamentales en la constitución de una red de servicios, pero hoy son solo subsidiarios de la lógica que pretenden sustituir dada la ausencia de planificaciones, de articulaciones, es decir la ausencia de una política pública que priorice el abordaje de los padecimientos subjetivos.

No se han abierto dispositivos, efectores o centros públicos comunitarios con posibilidad de abordaje de procesos asistenciales para la población con problemáticas graves en salud mental. El Estado de la Provincia de Santa Fe carece de instituciones para alojar las situaciones de consumo problemático, de vulneración de derechos de niños y adolescentes, las crisis subjetivas en la infancia, o instituciones que aborden la discapacidad; situaciones que terminan siendo derivadas al manicomio o a clínicas privadas, con convenios que amparan la derivación de los fondos públicos del Estado. 
Los dispositivos sustitutivos que actualmente funcionan en nuestra ciudad son experiencias muy valiosas que se sostienen fundamentalmente con la voluntad y la clínica artesanal de los trabajadores. En muy precarias condiciones en varios sentidos: salariales, contractuales, de salud, de insumos básicos, pero fundamentalmente la precariedad más importante radica en el modo en que tal o cual dispositivo se inserta (o no) en la red de salud y en la estructura administrativa de la cual se supone que depende.

Al ser escasos y al estar tan condicionados por esta precariedad el resultado de sus intervenciones y el acceso de los usuarios a estos es muy reducida. (AUGSBURGER AC; GERLERO SS; TABOADA E, 2016)

Sucede finalmente una especie de convivencia entre la lógica manicomial- que sigue hegemonizando el campo y concentrando la mayor parte del presupuesto- y los intentos de sustituirla con estos dispositivos. El resultado no es ni un empate, termina siendo una opción alternativa y no sustitutiva.

El riesgo es el de un modelo mixto, fracaso demostrado por muchos intentos de reforma y teorizado ampliamente por la experiencia italiana. Los dispositivos y prácticas alternativas conviven pacíficamente con el manicomio en tanto este se constituye en lugar de descarga y ocultamiento de todo aquello que resulta incoherente respecto a los códigos de interpretación e intervención de otras instituciones, es decir continúa administrando lo residual. Los riesgos más conocidos son la transinstitucionalización, la deshospitalización, puerta giratoria, achicamiento y más desasistencia (AUGSBURGER AC; GERLERO SS; TABOADA E., 2016)

El pasaje a lo sustitutivo creemos, no es ni la suma de dispositivos ni el cierre caprichoso de camas. Es fundamentalmente cualitativo respecto de la lógica que subvierte y genera una práctica de cuidado que sustituye.

Se hace necesario comprender que la desmanicomialización corre el riesgo de traducirse en desmantelamiento de instituciones o achicamiento del Estado; si es confundida con reducción de camas en los hospitales, del número de personal hospitalario, del número de internaciones, o un aumento de altas sin estrategias territoriales que contemplen las múltiples dimensiones de las problemáticas en salud mental.

El desmantelamiento del modelo manicomial no solo implica reformas estructurales dentro del sistema de salud, sino que también requiere de una profunda transformación de otros ámbitos necesarios para la restitución o armado de la vida cotidiana en una comunidad dada.

En el marco de la carencia de un plan de salud y las políticas mercantiles que rigen hoy el sentido de la economía en nuestro país, el resultado de todo esto es la Privatización de la Cronificación. 
Cronificación que el propio estado provoca con la ausencia de lugares públicos apropiados para alojar los padecimientos más emergentes que esta sociedad genera. Vulnerando aún más y transformándose en un Estado cómplice y partícipe de la proliferación del negocio privado de la discapacidad, de las clínicas de salud mental, de la industria farmacológica, de las instituciones religiosas, de las ONGs; todas instituciones que lucran con la salud de la población. Políticas planificadas que abren la puerta a mercados que avanzan sobre las prestaciones públicas que deben garantizar el derecho a la salud.

En este contexto, se torna urgente pensar en las instituciones que necesitamos para abordar las problemáticas de esta población; el Estado no puede seguir dependiendo de sus precarios convenios o acuerdos con el sector privado; no podemos confiarle a ellos el cuidado de los sujetos más vulnerados de nuestra sociedad; porque a ellos los mueve el negocio; en cambio en nuestra provincia, y en Argentina en general, estamos minados de cientos de trabajadores con un alto compromiso, formados y dispuestos a crear y sostener espacios transformadores.

La producción de registros, la creación de datos epidemiológicos y la valoración de los procesos de trabajo de quienes cotidianamente sostenemos nuestras prácticas asistenciales; podría generar una planificación de mayor eficacia, con presupuestos apropiados y acordes a las necesidades de la población; superando la sola acumulación de experiencias alternativas desprovistas de objetivos estratégicos; y permitiendo la creación de una red de salud integral que garantice el derecho a la salud con tratamientos centrados en lo comunitario.

Los sectores estatales ligados a la vivienda, al trabajo, a la seguridad social, o a la cultura tienen el desafío de la construcción de estrategias y redes que acompañen al cumplimiento de los objetivos y plazos anunciados en la Ley Nacional de Salud Mental y Adicciones. Se hace necesario el despliegue de una política pública intersectorial que permita la integración de los diversos sectores para generar propuestas a nivel territorial y comunitario, que aborden las problemáticas subjetivas no solo desde las lógicas del tratamiento, sino sobre todo desde las lógicas de la promoción y prevención de la salud.

Sostenemos como desafío: cerrar el manicomio, transformar su lógica, para abrir una perspectiva del cuidado en salud que garantice derechos y mejores condiciones de asistencia y de vida. 
En ese sentido nuestro planteo más que nunca debe ser abrir, y exigir junto con otros colectivos del campo el diseño coherente e integral de una política en salud mental que sustituya el manicomio sin desasistir.

Abrir en el sentido de ampliar lo público estatal, entendiendo a la salud como un derecho social esencial y a los sistemas públicos como genuinos redistribuidores del ingreso en favor de las mayorías de nuestros pueblos.

Abrir en el sentido del:

desarrollo de un sistema Único, Universal, integral e integrado, equitativo, calificado, eficiente y transparente de Seguridad Social y Salud pública financiado por rentas generales del Estado (...) desarrollado por trabajadores y trabajadoras sin condiciones precarizantes de trabajo y medio ambiente laboral. Implementando un plan de inversión hacia el sector, basado en la incorporación de personal, adecuación edilicia y tecnológica en los establecimientos públicos (IDEP, 2016)

Este diagnóstico que compartimos, se basa en una lectura desde la perspectiva de los trabajadores de la tensión inevitable que se genera en el marco de nuestras prácticas en salud pública y en el diseño de las mismas. El origen de nuestro análisis no es la academia sino el conflicto que se desprende de nuestro proceso de trabajo -condiciones de trabajo-y una ética de transformación que nos interpela e impacta en los servicios que ofrecemos -condiciones de asistencia-.

El acontecimiento político -como lo fue la constitución de la Red Latinoamericana de derechos humanos y salud mental (Declaración de Florianópolis, 2017), en el marco del Primer Encuentro Latinoamericano de Derechos Humanos y Salud Mental, así como también la generosa invitación a participar de esta publicación; nos conmueve y nos invita a seguir propiciando el encuentro de actores claves del campo popular, del campo universitario, del campo de los derechos humanos, de los trabajadores y de la comunidad, para la creación de herramientas colectivas que multipliquen experiencias transformadoras en el campo de la salud y generen resistencia a la avanzada neoliberal en nuestros países.

\section{Bibliografía}

AMARANTE, P. "Locos por la vida" Trayectoria de la reforma psiquiátrica en Brasil. $1^{\mathrm{a}}$ ed. Ciudad Autónoma de Buenos Aires: Asoc. Madre de Plaza de Mayo, 2006.

AUGSBURGER AC.; GERLERO SS; TABOADA E. (Comp.) Las políticas públicas en Salud Mental a cinco años de sanción de la Ley Nacional. Argentina: Editorial Instituto de la Salud Juan Lazarte, 2016. 
FERREYRA, J. y STOLKINER, A. El cambio es el retroceso. Que paso en Salud Mental durante el último año de gobierno. Diario Página 12. Suplemento Psicología, jueves 12 de enero. 2017 Argentina.

MIGUELES L. Exposición Seminario Una Mirada Internacional, Latinoamericana y Argentina sobre la CUS. Rosario, junio 2017. Texto inédito.

LAURELL, A. C. Revisando las politicas y discursos en salud en America Latina. Revista Medicina Social. Vol. 5, Numero 1. Marzo 2010. (www.medicinasocial.info)

ONOCKO CAMPOS, R. La planificación en el laberinto: un viaje hermenéutico. Buenos Aires: Ed. Lugar, (2007)

ROTELLI, F. Vivir sin manicomios. La experiencia de Trieste. Ciudad autónoma de Buenos Aires: Topia Editorial, 2014.

STOLKINER, A; COMES, Y; GARBUS, P. "Alcances y potencialidades de la Atención Primaria de la Salud en Argentina" Ciencia \& saúde Colectiva, 16 (6); 2807-2816. (2011)

\section{Documentos:}

CONISMA. Consejo Consultivo Honorario de salud mental y adicciones. Ley 26657. Actualidad de la implementación de la Ley Nacional de Salud Mental en Argentina.

Diciembre de 2016.

DECLARACION DE ALMA-ATA Conferencia Internacional sobre Atención Primaria de Salud, Alma-Ata, URSS, 6-12 de septiembre de 1978 DNU 908/2016. htpp://servicios.infoleg.gob. ar/infoleglnternet/anexos/260000-264999/264047/norma.htm

IDEP SALUD-ATE Argentina. CUS: Golpe al derecho a la salud, universal a la estabilidad de los trabajadores. Boletín campaña \#NO a la CUS. Argentina, 2016.

IDEP SALUD-ATE Argentina. Elementos Clave del Decreto de Necesidad y Urgencia 908/2016 que establece la CUS en Argentina - Documento Movimiento por el Derecho a la Salud - Argentina, 2017.

Investigación "Cruzar el muro" del CELS (Centro de Estudios Legales y Sociales) (2015) http://www.cels.org.ar/especiales/cruzarelmuro/ 
LEY NACIONAL DE SALUD MENTAL Y ADICCIONES No 26.657. Argentina (2010). http:/l www.msal.gov.ar/saludmental/images/stories/info-equipos/pdf/2013-og-26 ley-nacionalsalud-mental.pdf.

OMS. Informe sobre la salud en el mundo. "La financiación de los Sistemas de Salud. EI camino hacia la cobertura universal" (2010) http://www.who.int/mediacentre/factsheets/ fs395/es/

PLAN NACIONAL DE SALUD MENTAL. Argentina (2013) http://www.msal.gov.ar/ saludmental/images/stories/info-equipos/pdf/2013-10-29 plan-nacional-salud-mental.pdf

RESOLUCIÓN 1061-E/2017 https://www.boletinoficial.gob. ar/\#!DetalleNormaBusquedaRapida/168749/20170808/Resoluci\%C3\%B3n\%201061$\mathrm{E} \% 2 \mathrm{~F} 2017$

\section{Resumen}

Discutir "diagnósticos y desafíos" actuales desde el lugar de trabajadores; implica una lectura TRANSVERSAL de las prácticas en el campo de la salud mental y el conflicto que se genera en la tensión inevitable entre éstas y el diseño de las políticas. Abordar el cierre del manicomio es prioritario para avanzar en un modelo de atención con perspectiva de derechos que garantice condiciones a los procesos de asistencia en salud mental. Dado el avance de los procesos de reforma en nuestro país y en la región, hablar de cierre nos plantea hoy una encrucijada. Debemos ser cautelosos y al mismo tiempo audaces. Hoy hablar de cierre coagula el sentido neoliberal del corrimiento del Estado como garante de derechos. Por eso decimos "Cerrar el manicomio no es achicar el Estado". El plateo es abrir, y exigir el diseño de una política en salud mental q sustituya el manicomio sin desasistir.

Palabras clave: Cierre del manicomio- Privatizacion del Estado-Trabajadores-Políticas Publicas. 